\title{
The Worsening of Positional Mild Obstructive Sleep Apnea over Time Is Associated with an Increase in Body Weight: Impact on Blood Pressure and Autonomic Nervous System
}

\author{
Wei-Hsiu Chang ${ }^{a}$ Hsien-Chang Wu ${ }^{\text {b,c }}$ Chou-Chin Lan ${ }^{\text {d, e }}$ Yao-Kuang Wu ${ }^{\text {d, e }}$ \\ Mei-Chen Yang ${ }^{d, e}$ \\ aDepartment of Internal Medicine, Taipei Medical University-Shuang Ho Hospital, Ministry of Health and Welfare, \\ New Taipei, Taiwan; ${ }^{b}$ Department of Traditional Chinese Medicine, Taipei Tzu Chi Hospital, Buddhist Tzu Chi Medical \\ Foundation, New Taipei, Taiwan; 'School of Post-Baccalaureate Chinese Medicine, Tzu Chi University, Hualien, \\ Taiwan; ${ }^{d}$ Division of Pulmonary Medicine, Department of Internal Medicine, Taipei Tzu Chi Hospital, Buddhist Tzu \\ Chi Medical Foundation, New Taipei, Taiwan; ${ }^{e}$ School of Medicine, Tzu Chi University, Hualien, Taiwan
}

\section{Keywords}

Obstructive sleep apnea · Mild · Positional dependency .

Natural course $\cdot$ Blood pressure $\cdot$ Autonomic nervous system

\begin{abstract}
Background: Most patients with mild obstructive sleep apnea (OSA) are positional dependent. Although mild OSA worsens over time, no study has assessed the natural course of positional mild OSA. Objectives: The aim of this study was to evaluate the natural course of positional mild OSA, its most valuable progression predictor, and its impact on blood pressure (BP) and the autonomic nervous system (ANS). Methods: This retrospective observational cohort study enrolled 86 patients with positional mild OSA and 26 patients with nonpositional mild OSA, with a follow-up duration of $32.0 \pm 27.6$ months and $37.6 \pm 27.8$ months, respectively. Polysomnographic variables, BP, and ANS functions were compared between groups at baseline and after follow-up. Results: In patients with positional mild OSA after follow-up, the apnea/hypopnea index (AHI) increased $(9.1 \pm$ $3.3 / \mathrm{h}$ vs. $22.0 \pm 13.2 / \mathrm{h}, p=0.000$ ), as did the morning systolic BP $(126.4 \pm 13.3 \mathrm{~mm} \mathrm{Hg}$ vs. $130.4 \pm 15.9 \mathrm{~mm} \mathrm{Hg}, p=$ $0.011)$, and the sympathetic activity $(49.4 \pm 12.3 \%$ vs. $55.3 \pm$
\end{abstract}

karger@karger.com www.karger.com/res

Karger"

GOPEN ACCESS
(C) 2021 The Author(s)

Published by S. Karger AG, Basel

This is an Open Access article licensed under the Creative Commons Attribution-NonCommercial-4.0 International License (CC BY-NC) (http://www.karger.com/Services/OpenAccessLicense), applicable to the online version of the article only. Usage and distribution for commercial purposes requires written permission.
$13.1 \%, p=0.000)$, while the parasympathetic activity decreased $(50.6 \pm 12.3 \%$ vs. $44.7 \pm 13.1 \%, p=0.000)$. The body mass index changes were the most important factor associated with $\mathrm{AHI}$ changes among patients with positional mild OSA (Beta $=0.259$, adjust $R^{2}=0.056, p=0.016,95 \%$ confidence interval 0.425 and 3.990). The positional dependency disappeared over time in $66.3 \%$ of patients with positional mild OSA while $69.2 \%$ of patients with nonpositional mild OSA retained nonpositional. Conclusions: In patients with positional mild OSA, disease severity, BP, and ANS regulation worse over time. Increased weight was the best predictor for its progression and the loss of positional dependency. Better treatments addressing weight control and consistent follow-up are needed for positional mild OSA.

$$
\begin{aligned}
& \text { C } 2021 \text { The Author(s) } \\
& \text { Published by S. Karger AG, Basel }
\end{aligned}
$$

\section{Introduction}

Obstructive sleep apnea (OSA) is a significant public health problem, given its association with cardiovascular comorbidities [1-6]. OSA severity is usually graded according to the apnea/hypopnea index (AHI) as follows: mild for AHI 5-14.9/h, moderate for AHI 15-29.9/h, and severe for 
AHI $>30 / h$. Treatment for OSA is generally based on AHI, symptoms, and cardiovascular complications. Generally, conservation strategies including weight reduction, sedatives or alcohol avoidance, and exercise were also suggested regardless of OSA severity. Specific treatments for mild OSA are recommended if patients have symptoms and/or cardiovascular comorbidities, including continuous positive airway pressure (CPAP), mandibular advanced device, surgical therapy, and positional therapy (PT) for patients with AHI improvement in the nonsupine sleep position [4, 7-9]. Long-term CPAP compliance is low in mild OSA patients [10]. Although mandibular advanced devices are effective for mild OSA and the report subjected long-term compliance satisfying [4], there are no sufficient studies assessing by objective methods the real compliance of this treatment. Surgical treatment has variable efficacy; however, it is highly selected for a minority of mild OSA patients [4]. Most patients with mild OSA show AHI improvement in the supine sleep position (positional mild OSA) [11], and commercially available positional control devices have been proved effective to treat positional mild OSA [9]. Positional mild OSA patients are usually advised PT only. However, PT compliance is difficult to monitor. In clinical practice, most patients with mild OSA do not receive any specific treatment [12].

Mild OSA has been shown to progress over time [1214], and active treatment for mild OSA is known to improve OSA severity and blood pressure (BP) control [5]. Since the majority of mild OSA is positional dependent (PP) [11], we hypothesized that positional mild OSA also worsens over time. There has been no study of the natural course of positional mild OSA. This study focused on the natural course of positional mild OSA, the predicting factors of the disease progression, and the impacts of disease progression on cardiovascular complications and sleep quality.

\section{Materials and Methods}

\section{Participants}

This was a retrospective observational cohort study. The inclusion criteria were (1) age 20-75 years old, (2) mild OSA with AHI $5-14.9 / \mathrm{h},(3)$ a raw PSG recording at baseline and after follow-up, (4) a PSG recording for $\geq 30 \mathrm{~min}$ in both supine and nonsupine positions, and (5) not receiving any active treatments during the follow-up period, including CPAP, surgical therapy, mandibular advancement device, PT, or body weight (BW) reduction surgery. The exclusion criteria were ( 1 ) AHI $<5 /$ h or AHI $\geq 15 / \mathrm{h}$ (2) congestive heart failure, unstable chronic obstructive pulmonary disease and/or asthma, severely impaired renal and liver function (shown in Fig. 1). A total of 396 patients from January 2007 to December

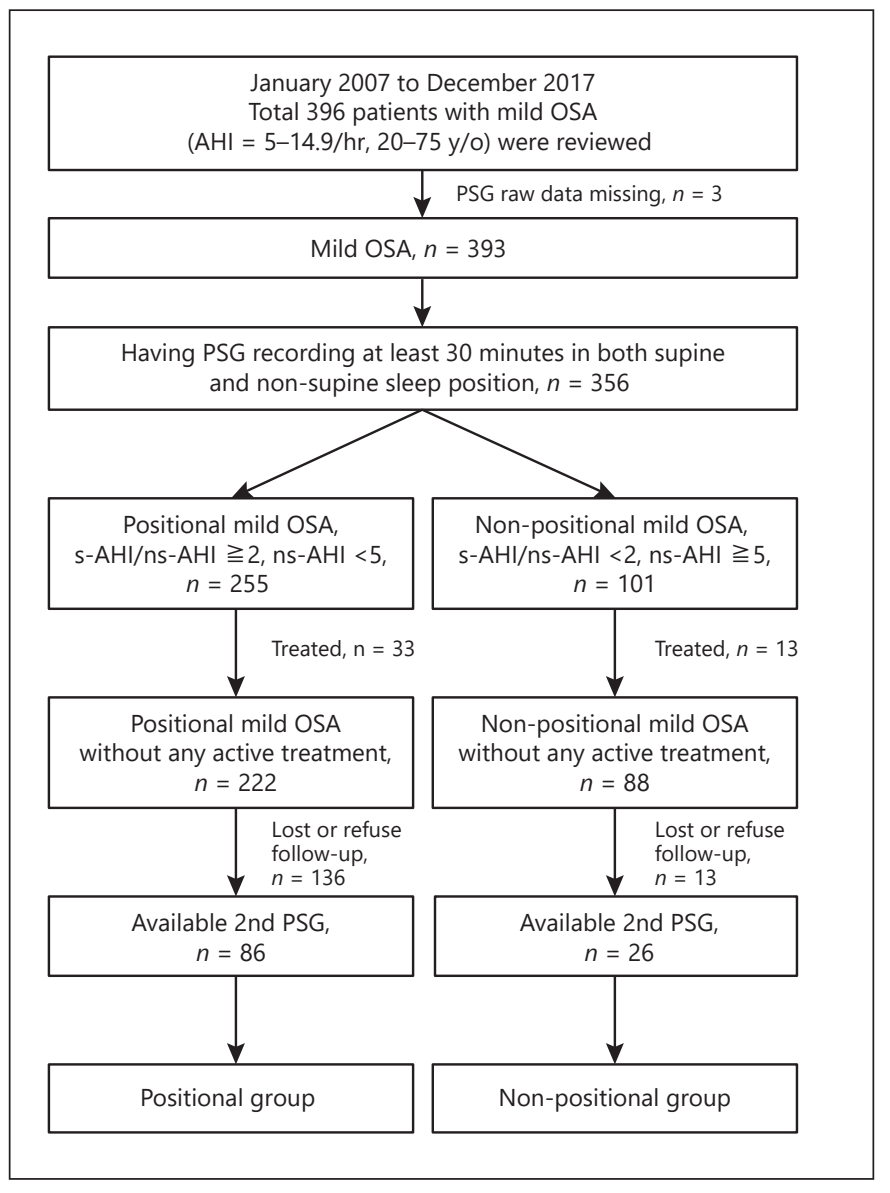

Fig. 1. Study flowchart.

2017 were reviewed but 3 patients' raw PSG data were missing. Among the remaining 393 patients, 356 patients had a PSG recording $\geq 30 \mathrm{~min}$ in both supine and nonsupine positions. Among 356 patients, 255 had normalized AHI in the nonsupine position (ns$\mathrm{AHI}<5 / \mathrm{h}$ ), and the AHI ratio in the supine position (s-AHI) to ns-AHI $\geq 2$ was defined to the positional mild OSA (PP group), and the other 101 patients who did not meet the abovementioned criteria were allocated to the nonpositional mild OSA (NPP group). After excluding patients with active treatment during the followup (33 for PP group and 13 for NPP group) and patients losing or refusing the follow-up (136 for PP group and 62 in NPP group), 86 patients in the PP group and 26 patients in the NPP group had a second PSG examination after the follow-up and were included in the final analysis. The follow-up duration was $32.0 \pm 27.6$ months for the PP group and $37.6 \pm 27.8$ months for the NPP group.

This study was approved by the Institutional Review Board of Taipei Tzu Chi Hospital (Protocol No. 07-XD-049). The requirement for informed consent was waived because of the retrospective design.

\section{Anthropometric Characteristics}

We collected information regarding age, gender, BW, body height, body mass index (BMI), smoking status, alcohol consumption status, and underlying comorbidities (diabetes mellitus, hy- 
Table 1. Baseline characteristics of patients with positional and nonpositional mild OSA

\begin{tabular}{|c|c|c|c|}
\hline & $\begin{array}{l}\text { Positional } \\
(n=86)\end{array}$ & $\begin{array}{l}\text { Nonpositional } \\
(n=26)\end{array}$ & $p$ value \\
\hline \multicolumn{4}{|l|}{ Characteristics } \\
\hline \multicolumn{4}{|l|}{ Gender } \\
\hline Male, $n(\%)$ & $57(66.5)$ & $18(69.2)$ & 0.779 \\
\hline Female, $n(\%)$ & $29(33.7)$ & $8(30.8)$ & \\
\hline Age, years old & $54.3 \pm 13.6$ & $54.5 \pm 10.6$ & 0.912 \\
\hline \multicolumn{4}{|l|}{ Underlying diseases } \\
\hline Diabetes mellitus, $n(\%)^{\S}$ & $22(25.6)$ & $4(15.4)$ & 0.427 \\
\hline Hypertension, $n(\%)$ & $35(40.7)$ & $12(46.2)$ & 0.621 \\
\hline Coronary artery disease, $n(\%)^{\S}$ & $9(10.5)$ & $2(7.7)$ & 1.000 \\
\hline Cerebrovascular accident, $n(\%)^{\S}$ & $2(2.3)$ & $1(3.8)$ & 0.551 \\
\hline Chronic obstructive airway disease, $n(\%)$ & $35(40.7)$ & $11(42.3)$ & 0.884 \\
\hline Smoking, $n(\%)$ & $29(33.7)$ & $11(42.3)$ & 0.423 \\
\hline Alcohol, $n(\%)^{\S}$ & $9(10.5)$ & $5(10.2)$ & 0.308 \\
\hline $\mathrm{BH}, \mathrm{cm}$ & $165.1 \pm 8.8$ & $165.5 \pm 10.2$ & 0.847 \\
\hline $\mathrm{BW}, \mathrm{kg}$ & $73.1 \pm 14.4$ & $77.3 \pm 14.4$ & 0.199 \\
\hline $\mathrm{BMI}, \mathrm{kg} / \mathrm{m}^{2}$ & $26.8 \pm 4.6$ & $28.1 \pm 3.7$ & 0.149 \\
\hline Follow-up duration, months & $32.0 \pm 27.6$ & $37.6 \pm 27.8$ & 0.375 \\
\hline
\end{tabular}

Categorical variables are presented as counts (\%) and were analyzed using Pearson's $\chi^{2}$ test (large sample, $n>5$ ). Continuous variables were presented as mean \pm standard deviation and were analyzed using the independent-sample $t$ test. ${ }^{\S}$ Fisher's exact test (small sample, $n \leq 5$ ). BH, body height; BW, body weight; BMI, body mass index; OSA, obstructive sleep apnea.

pertension, cardiovascular accident, coronary artery disease, or chronic obstructive airway diseases [chronic obstructive pulmonary disease and/or asthma]).

\section{BP Measurements}

Trained sleep technicians performed routine BP measurements at the sleep center on the right or left arm using a standard automatic sphygmomanometer (Welch Allyn Vital Signs Monitor 300 Series), calibrated annually by the manufacturer and regularly validated using manual measurements with a standard mercury sphygmomanometer. Evening BP measurements were obtained before sleep onset, after setting up the PSG apparatus, and after the patients had remained in the supine position at least $20 \mathrm{~min}$. Morning BP measurements were obtained immediately after waking up, with the patient still in the supine position and using the PSG apparatus. Two consecutive BP measurements were performed on each occasion, and their average was obtained as the recorded measurement.

\section{Polysomnography Recordings}

Trained sleep technicians performed standard overnight PSG. The items recorded included the position, oxygen desaturation, snoring status, and airflow through the nose and mouth (as determined using a thermistor). The body position was identified using a trunk-position sensor. Each PSG recording session lasted at least $6 \mathrm{~h}$. Each 30-s epoch was manually scored by trained sleep technicians and rechecked by a sleep specialist. Sleep stage and respiratory events were rescored according to the 2012 AASM criteria.

\section{Excessive Daytime Sleepiness}

Excessive daytime sleepiness was assessed using the Chinese version of the Epworth Sleepiness Scale before the overnight PSG [15].

\section{Autonomic Nervous System Activity Measurements}

Heart rate variability was measured as a function of the autonomic nervous system (ANS). After reimporting raw PSG data to the software of ProFusion PSG 4.0 Sleep Diagnostic system (Somte PSG, Compumedics, Abbotsford, VIC, Australia), heart rate variability measurements were obtained by analyzing the electrocardiogram montage using the Somte ECG Analysis Option Software (ECG Addon for ProFusion PSG 4, Version 1.0 Beta) at 5-min intervals during sleep time. Frequency-domain methods were based on power spectral density and provide basic information regarding the distribution of power (i.e., variance) as a function of frequency. Two main spectral components are distinguished in a spectrum calculated from short-term recordings obtained at 5-min intervals during the sleep time (1) high frequency (HF, from 0.15 to $0.4 \mathrm{~Hz}$ ) and (2) low frequency (LF, from 0.04 to $0.15 \mathrm{~Hz}$ ). The spectral components were expressed as normalized units $(\mathrm{nu})$ to minimize the effect of changes in the total power on the values of the LF and HF components. The calculation was performed using the following formula (absolute power of the component $) \times 100 /(\mathrm{LF}+\mathrm{HF})$. The LFnu and HFnu represent the sympathetic and parasympathetic activity, respectively. The LF/HF ratio indicated the overall ANS regulation. The LFnu, HFnu, and LF/HF ratio recordings were collected at 5-min intervals during the sleep time and their average values used for the final analysis [16]. 
Table 2. Comparison of BP, polysomnographic variables, and autonomic activity at baseline and after follow-up within each group, and between 2 groups at baseline and after follow-up

\begin{tabular}{|c|c|c|c|c|c|c|c|c|}
\hline & \multicolumn{3}{|c|}{ PP group $(n=86)$} & \multicolumn{3}{|c|}{ NPP group $(n=26)$} & \multicolumn{2}{|c|}{$\begin{array}{l}\text { Difference between } \\
\text { PP and NPP groups }\end{array}$} \\
\hline & baseline & after & $p$ value $^{\mathrm{a}}$ & baseline & after & $p$ value $^{\mathrm{a}}$ & $\begin{array}{l}\text { baseline } \\
p \text { value }^{\mathrm{b}}\end{array}$ & $\begin{array}{l}\text { follow-up } \\
p \text { value }^{c}\end{array}$ \\
\hline $\mathrm{BW}, \mathrm{kg}$ & $73.1 \pm 14.4$ & $74.1 \pm 14.9$ & 0.015 & $77.3 \pm 14.4$ & $77.8 \pm 13.9$ & 0.426 & 0.199 & 0.265 \\
\hline $\mathrm{BMI}, \mathrm{kg} / \mathrm{m}^{2}$ & $26.8 \pm 4.6$ & $27.2 \pm 4.8$ & 0.006 & $28.1 \pm 3.7$ & $28.3 \pm 3.5$ & 0.397 & 0.149 & 0.223 \\
\hline Morning sBP, mm Hg & $126.4 \pm 13.3$ & $130.4 \pm 15.9$ & 0.011 & $133.8 \pm 15.1$ & $137.7 \pm 15.5$ & 0.243 & 0.031 & 0.043 \\
\hline Morning dBP, $\mathrm{mm} \mathrm{Hg}$ & $79.8 \pm 11.8$ & $79.7 \pm 12.0$ & 0.911 & $83.3 \pm 10.6$ & $84.3 \pm 11.5$ & 0.727 & 0.158 & 0.081 \\
\hline ESS & $8.9 \pm 4.2$ & $9.7 \pm 4.7$ & 0.078 & $9.8 \pm 4.8$ & $10.5 \pm 5.4$ & 0.360 & 0.410 & 0.474 \\
\hline \multicolumn{9}{|l|}{ Polysomnographic variables } \\
\hline Sleep efficiency, \% & $76.2 \pm 15.7$ & $78.4 \pm 13.2$ & 0.170 & $72.9 \pm 16.6$ & $72.0 \pm 13.8$ & 0.816 & 0.379 & 0.043 \\
\hline N1 sleep, \% & $14.4 \pm 6.3$ & $17.9 \pm 7.9$ & 0.001 & $14.5 \pm 7.2$ & $19.8 \pm 10.4$ & 0.009 & 0.981 & 0.278 \\
\hline AHI, /h & $9.1 \pm 3.3$ & $22.0 \pm 13.2$ & 0.000 & $11.3 \pm 3.2$ & $23.6 \pm 16.0$ & 0.000 & 0.004 & 0.650 \\
\hline s-AHI, /h & $18.0 \pm 13.1$ & $31.5 \pm 19.8$ & 0.000 & $19.1 \pm 13.3$ & $35.6 \pm 21.8$ & 0.000 & 0.708 & 0.393 \\
\hline ns-AHI, /h & $2.0 \pm 2.6$ & $11.6 \pm 13.7$ & 0.000 & $7.8 \pm 2.1$ & $11.1 \pm 10.0$ & 0.092 & 0.000 & 0.842 \\
\hline $\mathrm{DI}, / \mathrm{h}$ & $6.9 \pm 4.6$ & $15.9 \pm 12.4$ & 0.000 & $7.7 \pm 3.4$ & $15.8 \pm 9.6$ & 0.000 & 0.307 & 0.975 \\
\hline $\mathrm{SaO}_{2}$ baseline, $\%$ & $94.8 \pm 1.4$ & $94.7 \pm 1.4$ & 0.595 & $94.6 \pm 1.6$ & $94.3 \pm 1.5$ & 0.387 & 0.602 & 0.330 \\
\hline $\mathrm{SaO}_{2}$ mean, $\%$ & $94.1 \pm 1.7$ & $93.9 \pm 1.5$ & 0.337 & $93.8 \pm 1.9$ & $93.5 \pm 1.9$ & 0.321 & 0.504 & 0.261 \\
\hline $\mathrm{SaO}_{2}$ nadir, $\%$ & $83.6 \pm 7.0$ & $82.9 \pm 6.0$ & 0.451 & $83.9 \pm 5.2$ & $81.7 \pm 6.0$ & 0.076 & 0.781 & 0.346 \\
\hline \%TIB with $\mathrm{SaO}_{2}<90 \%, \%$ & $4.5 \pm 11.5$ & $6.5 \pm 10.6$ & 0.129 & $3.4 \pm 3.5$ & $8.5 \pm 13.6$ & 0.075 & 0.443 & 0.495 \\
\hline \multicolumn{9}{|l|}{ Autonomic nervous activity } \\
\hline Sympathetic, \% & $49.4 \pm 12.3$ & $55.3 \pm 13.1$ & 0.000 & $48.4 \pm 12.6$ & $59.0 \pm 12.0$ & 0.000 & 0.740 & 0.187 \\
\hline Parasympathetic, $\%$ & $50.6 \pm 12.3$ & $44.7 \pm 13.1$ & 0.000 & $51.6 \pm 12.6$ & $41.0 \pm 12.0$ & 0.000 & 0.740 & 0.187 \\
\hline
\end{tabular}

sBP, systolic blood pressure; $\mathrm{dBP}$, diastolic blood pressure; REM, rapid eye movement; AI, arousal index; AHI, apnea/hypopnea index; s-AHI, AHI in supine position; ns-AHI, AHI in nonsupine position; DI, desaturation index; $\mathrm{SaO}_{2}$, oxygen saturation; TIB, time in bed; BP, blood pressure; BW, body weight; PP, positional dependent; NPP, nonpositional dependent; ESS, Epworth sleepiness scale. Continuous variables were presented as mean \pm standard deviation. Bold indicates statistical significance at $p<0.05$ with 2 tails. ${ }^{a}$ Comparisons between baseline and after follow-up within each group using paired-samples $t$ test. ${ }^{\mathrm{b}}$ Comparisons between 2 groups at baseline using independent-samples $t$ test. ${ }^{\mathrm{C}}$ Comparisons between 2 groups after follow-up using independent-samples $t$ test.

\section{Statistical Analysis}

Categorical variables were presented as counts (\%) and analyzed using Pearson's $\chi^{2}$ test (large samples, $n>5$ ) or Fisher's exact test (for small samples, $n \leq 5$ ). Continuous variables were presented as mean \pm standard deviation. The independent-sample $t$ test was used for between-group comparisons at baseline and at follow-up. The paired-samples $t$ test was used for within-group comparisons between baseline and the follow-up. To estimate relevant factors affecting AHI and BP changes, univariate analysis (independent-sample $t$ test for binary independent variables, and linear regression for continuous independent variables) was performed. All significant factors identified in univariable analyses, except for those which showed collinearity with other variables, were entered into a stepwise multivariate linear regression model to assess the simultaneous contribution of these factors. Univariate analysis us- ing binary logistic regression was used to evaluate factors predicting the preservation or loss of positional dependency over time in PP group. All statistical assessments were 2 -sided and evaluated at the $p<0.05$ level of statistical significance. Statistical analyses were performed with statistics software (IBM SPSS version 24 for Windows, IBM Corp., Armonk, New York, NY, USA).

\section{Results}

Table 1 shows between-group comparisons at baseline. There was no significant between-group difference in the age, gender, comorbidities, smoking status, alcohol 
Table 3. Multivariable stepwise linear regression analysis of factor significantly associated with the changes of AHI in univariate analysis among patients with positional mild obstructive sleep apnea, patients with nonpositional mild obstructive sleep apnea, and patients with mild obstructive sleep apnea, respectively

\begin{tabular}{|c|c|c|c|c|c|}
\hline \multirow[t]{2}{*}{ Model } & Unstandardized coefficients & $\begin{array}{l}\text { Standardized } \\
\text { coefficients }\end{array}$ & $t$ & Sig & $95 \%$ CI for $B$ \\
\hline & std. error & beta & & & lower bound upper bound \\
\hline
\end{tabular}

Among patients with positional mild OSA, model based on $\Delta \mathrm{BMI}$, with adjust $R^{2}=0.056, p=0.016$, excluded independent variables including age at baseline, age at follow-up, follow-up duration, and $\triangle \mathrm{BW}$

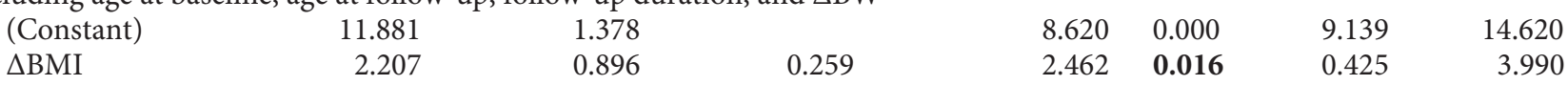

Among patients with nonpositional mild OSA, model based on $\triangle \mathrm{BMI}$, with adjust $R 2=0.040, p=0.019$, excluded independent variables including follow-up duration, $\mathrm{BW}$ at baseline, and $\triangle \mathrm{BW}$
(Constant)
11.940
1.271
$2.063 \quad 0.869$
$9.395 \quad 0.000$
9.421
14.458
$\triangle \mathrm{BMI}$

follow-up duration

variables including, $\triangle \mathrm{BW}$ and follow-up duration

\begin{tabular}{|c|c|c|c|c|c|c|c|}
\hline (Constant) & -1.854 & 6.375 & & -0.291 & 0.772 & -14.489 & 10.782 \\
\hline$\Delta \mathrm{BMI}$ & 2.193 & 0.856 & 0.235 & 2.562 & 0.012 & 0.496 & 3.889 \\
\hline$I$ at baseline & 0.186 & 0.084 & 0.202 & 2.206 & 0.029 & 0.019 & 0.352 \\
\hline
\end{tabular}

OSA, obstructive sleep apnea; BMI, body mass index; BW, body weight; AHI, apnea/hypopnea index; CI, confidence interval. Bold indicates statistical significance at $p<0.05$ with 2 tails.

Table 4. Stepwise linear regression analysis of factor most significantly associated with $\Delta$ morning sBP in univariate analysis among patient with mild positional OSA

\begin{tabular}{|c|c|c|c|c|c|c|c|}
\hline & \multicolumn{2}{|c|}{$\begin{array}{l}\text { Unstandardized } \\
\text { coefficients }\end{array}$} & \multirow{2}{*}{$\begin{array}{l}\text { Standardized } \\
\text { coefficients } \\
\text { beta }\end{array}$} & \multirow[t]{2}{*}{$t$} & \multirow[t]{2}{*}{ Sig } & \multicolumn{2}{|l|}{$95 \%$ CI for B } \\
\hline & $B$ & std. error & & & & lower bound & upper bound \\
\hline (Constant) & -12.085 & 6.502 & & -1.859 & 0.067 & -25.015 & 0.846 \\
\hline Age at follow-up & 0.281 & 0.111 & 0.266 & 2.526 & 0.013 & 0.060 & 0.502 \\
\hline
\end{tabular}

Dependent variable: $\Delta$ morning sBP. Excluded independent variable: age at baseline. Model based on age at follow-up, with adjust $R 2=0.060, p=0.013$. Bold indicates statistical significance at $p<0.05$ with 2 tails. sBP, systolic blood pressure; CI, confidence interval; OSA, obstructive sleep apnea.

consumption status, BW, body height, BMI, and the follow-up duration. Table 2 shows between-group comparisons at baseline and after follow-up, as well as withingroup comparisons after the follow-up.

At baseline, the NPP group had significantly higher morning systolic blood pressure (sBP) $(126.4 \pm 13.3 \mathrm{~mm}$ $\mathrm{Hg}$ vs. $133.8 \pm 15.1 \mathrm{~mm} \mathrm{Hg}, p=0.031)$, higher AHI (9.1 $\pm 3.3 / \mathrm{h}$ vs. $11.3 \pm 3.2 / \mathrm{h}, p=0.004)$, and higher ns-AHI $(2.0$ $\pm 2.6 / \mathrm{h}$ vs. $7.8 \pm 2.1 / \mathrm{h}, p=0.000)$ than the PP group. After the follow-up, the NPP group had significantly higher evening sBP $(129.1 \pm 16.3 / \mathrm{h}$ vs. $137.5 \pm 19.0 / \mathrm{h}, p=0.048)$ and higher morning sBP $(130.4 \pm 15.9 / \mathrm{h}$ vs. $137.7 \pm$ $15.5 / \mathrm{h}, p=0.043)$ than the PP group.
After follow-up, the PP group had significantly increased BMI ( $26.8 \pm 4.6$ vs. $\left.27.2 \pm 4.8 \mathrm{~kg} / \mathrm{m}^{2}, p=0.006\right)$, increased morning sBP $126.4 \pm 13.3 \mathrm{~mm} \mathrm{Hg}$ versus 130.4 $\pm 15.9 \mathrm{~mm} \mathrm{Hg}, p=0.011$ ), and increased arousal index $(13.6 \pm 6.7 / \mathrm{h}$ vs. $20.8 \pm 12.2 / \mathrm{h}, p=0.000)$. After follow-up, both groups had increased N1 sleep stage ( $14.4 \pm 6.3 \%$ vs. $17.9 \pm 7.9 \%, p=0.001$ for PP group and $14.5 \pm 7.2 \%$ vs. $19.8 \pm 10.4 \%, p=0.009$ for NPP group), increased AHI $(9.1 \pm 3.3 / \mathrm{h}$ vs. $22.0 \pm 13.2 / \mathrm{h}, p=0.000$ for PP group; 11.3 $\pm 3.2 / \mathrm{h}$ vs. $23.6 \pm 16.0 / \mathrm{h}, p=0.000$ for NPP group), increased desaturation index $(6.9 \pm 4.6 / \mathrm{h}$ vs. $15.9 \pm 12.4 / \mathrm{h}$, $p=0.000$ for PP group; $7.7 \pm 3.4 / \mathrm{h}$ vs. $15.8 \pm 9.6 / \mathrm{h}, p=$ 0.000 for NPP group), increased sympathetic activity 
Table 5. Positional dependency and severity conversion after follow-up

\begin{tabular}{llcc}
\hline & $\begin{array}{l}\text { Positional } \\
(n=86)\end{array}$ & $\begin{array}{l}\text { Nonpositional } \\
(n=26)\end{array}$ & $p$ value \\
\hline $\begin{array}{l}\text { Positional dependency conversion } \\
\quad \text { Positional, } n(\%)\end{array}$ & $29(33.7)$ & $8(30.8)$ & 0.779 \\
$\quad$ Nonpositional, $n(\%)$ & $57(66.3)$ & $18(69.2)$ & \\
Overall AHI & & $4(15.4)$ & 0.083 \\
$\quad$ Improvement, $n(\%)$ & $82(95.3)$ & $22(84.6)$ & \\
$\quad$ Progression, $n(\%)$ & $54(62.8)$ & $20(76.9)$ & 0.182 \\
Moderate to severe conversion & $32(37.2)$ & $6(23.1)$ & \\
$\quad \begin{array}{l}\text { Conversion, } n(\%) \\
\text { No conversion, } n(\%)\end{array}$ & & & \\
\hline
\end{tabular}

Categorical variables are presented as counts (\%) and were compared using Pearson's $\chi^{2}$ test (large sample, $n>5$ ). ${ }^{\mathfrak{S}}$ Fisher's exact test (small sample, $\left.n \leq 5\right)$. AHI, apnea/hypopnea index.

Table 6. The factors predicting the increased N1 sleep stage in both groups after follow-up

\begin{tabular}{|c|c|c|c|c|c|c|c|}
\hline & \multicolumn{2}{|c|}{$\begin{array}{l}\text { Unstandardized } \\
\text { coefficients }\end{array}$} & \multirow{2}{*}{$\begin{array}{l}\text { Standardized } \\
\text { coefficients } \\
\text { beta }\end{array}$} & \multirow[t]{2}{*}{$t$} & \multirow[t]{2}{*}{ Sig } & \multicolumn{2}{|l|}{$95 \%$ CI for $B$} \\
\hline & $B$ & std. error & & & & lower bound & upper bound \\
\hline \multicolumn{8}{|l|}{ PP group } \\
\hline (Constant) & -2.249 & 1.137 & & -1.979 & 0.051 & -4.510 & 0.012 \\
\hline$\Delta \mathrm{BMI}$ & 0.343 & 0.505 & 0.065 & 0.679 & 0.499 & -0.663 & 1.349 \\
\hline$\Delta \mathrm{AHI}$ & 0.334 & 0.060 & 0.535 & 5.541 & 0.000 & 0.214 & 0.454 \\
\hline \multicolumn{8}{|l|}{ NPP group } \\
\hline (Constant) & 1.180 & 2.714 & & 0.435 & 0.668 & -4.463 & 6.823 \\
\hline$\triangle \mathrm{BMI}$ & -0.979 & 1.387 & -0.121 & -0.706 & 0.488 & -3.863 & 1.905 \\
\hline$\Delta \mathrm{AHI}$ & 0.400 & 0.104 & 0.640 & 3.845 & 0.001 & 0.184 & 0.616 \\
\hline
\end{tabular}

$\Delta$, difference of the value at baseline and the value after follow-up. Bold indicates statistical significance at $p<$ 0.05 with 2 tails. BMI, body mass index; AHI, apnea/hypopnea index; PP, positional dependent; NPP, nonpositional dependent; CI, confidence interval.

$(49.4 \pm 12.3 \%$ vs. $55.3 \pm 13.1 \%, p=0.000$ for PP group; $48.4 \pm 12.6 \%$ vs. $59.0 \pm 12.0 \%, p=0.000$ for NPP group), decreased parasympathetic activity $(50.6 \pm 12.3 \%$ vs. 44.7 $\pm 13.1 \%, p=0.000$ for PP group; $51.6 \pm 12.6 \%$ vs. $41.0 \pm$ $12.0 \%, p=0.000$ for NPP group), and increased overall ANS activity $(1.13 \pm 0.72$ vs. $1.49 \pm 0.97, p=0.000$ for PP group; $1.08 \pm 0.66$ vs. $1.78 \pm 1.43, p=0.000$ for NPP group).

Univariable analysis revealed that AHI changes $(\triangle \mathrm{AHI})$ were positively correlated with BMI changes $(\triangle \mathrm{BMI}), \mathrm{BW}$ changes $(\Delta \mathrm{BW})$, follow-up duration, age at baseline, and age at follow-up, but only the $\triangle \mathrm{BMI}$ was the most relevant independent factor associated with the $\triangle \mathrm{AHI}$ among PP group in stepwise multivariate linear regression analysis (Beta $=0.259$, adjust $R^{2}=0.056, p=$ 0.016 , with $95 \%$ confidence interval 0.425 and 3.990) (Ta- ble 3). A model for the $\Delta \mathrm{AHI}$ was $\Delta \mathrm{AHI}=\Delta \mathrm{BMI} \times 2.2067$ +11.881 .

Univariable analysis revealed that increased morning sBP was associated with age at baseline and age at followup, but the most relevant independent factor was age at follow-up (Beta $=0.266$, adjust $R^{2}=0.060, p=0.013$, with $95 \%$ confidence interval 0.060 and 0.502 ) (Table 4).

As showed in Table 5, after the follow-up, the prevalence of positional dependency in each group was not statistically different ( $66.3 \%$ vs. $69.2 \%, p=0.779)$, while OSA severity worsened in both groups (overall AHI progression: $95.3 \%$ in PP group vs. $84.6 \%$ in NPP group, $p=0.083$ and moderate to severe conversion: $37.2 \%$ in PP group vs. $23.1 \%$ in NPP group, $p=0.182$ ).

In both groups, the increased N1 sleep stage was positively correlated to the increased AHI, but not the in- 
Table 7. Factors predicting the preservation or loss of positional dependency in PP group

\begin{tabular}{|c|c|c|c|c|c|c|c|c|}
\hline \multirow[t]{2}{*}{ Factors } & \multirow[t]{2}{*}{$B$} & \multirow[t]{2}{*}{ SE } & \multirow[t]{2}{*}{ Wald } & \multirow[t]{2}{*}{ df } & \multirow[t]{2}{*}{ Sig } & \multirow[t]{2}{*}{$\operatorname{Exp}(B)$} & \multicolumn{2}{|c|}{$95 \%$ CI for $\operatorname{Exp}(B)$} \\
\hline & & & & & & & lower & upper \\
\hline \multicolumn{9}{|c|}{ Binary logistic regression of factors predicting the lost or preservation of positional dependency in PP group } \\
\hline Gender & 0.281 & 0.477 & 0.346 & 1 & 0.556 & 1.324 & 0.520 & 3.373 \\
\hline Alcohol & -0.019 & 0.747 & 0.001 & 1 & 0.979 & 0.981 & 0.227 & 4.241 \\
\hline Smoke & -0.183 & 0.488 & 0.141 & 1 & 0.707 & 0.833 & 0.320 & 2.167 \\
\hline Age at baseline & -0.033 & 0.018 & 3.430 & 1 & 0.064 & 0.967 & 0.934 & 1.002 \\
\hline Age at follow-up & -0.032 & 0.018 & 2.975 & 1 & 0.085 & 0.969 & 0.935 & 1.004 \\
\hline Follow-up duration & 0.008 & 0.009 & 0.814 & 1 & 0.367 & 1.008 & 0.991 & 1.026 \\
\hline BW at baseline & 0.035 & 0.018 & 3.764 & 1 & 0.052 & 1.036 & 1.000 & 1.073 \\
\hline BW at follow-up & 0.038 & 0.018 & 4.291 & 1 & 0.038 & 1.038 & 1.002 & 1.076 \\
\hline Changes of BW & 0.046 & 0.060 & 0.597 & 1 & 0.440 & 1.047 & 0.932 & 1.177 \\
\hline BMI at baseline & 0.104 & 0.057 & 3.271 & 1 & 0.070 & 1.109 & 0.991 & 1.241 \\
\hline BMI at follow-up & 0.111 & 0.058 & 3.730 & 1 & 0.053 & 1.118 & 0.998 & 1.251 \\
\hline Changes of BMI & 0.114 & 0.162 & 0.493 & 1 & 0.483 & 1.121 & 0.815 & 1.540 \\
\hline $\mathrm{AHI}$ at baseline & 0.097 & 0.073 & 1.775 & 1 & 0.183 & 1.102 & 0.955 & 1.272 \\
\hline
\end{tabular}

BW, body weight; BMI, body mass index; AHI, apnea/hypopnea index; PP, positional dependent; CI, confidence interval. Bold indicates statistical significance at $p<0.05$ with 2 tails.

creased BMI (Table 6). Only the BW at follow-up was positively associated with the loss of positional dependency in the PP group (Table 7).

\section{Discussion}

We observed that, over time, positional mild OSA worsens and is associated with increasing cardiovascular complications, while the positional dependency disappeared. Increasing weight was associated with disease progression and the loss of positional dependency. Increasing $\mathrm{BP}$ was positively related with age at follow-up.

The prevalence of positional dependency varies according to different definitions and depends on age, BMI, and AHI $[7,11,17,18]$. Positional OSA was previously defined as an $\mathrm{AHI} \geq 5 / \mathrm{h}$ with s-AHI/ns- $\mathrm{AHI}$ ratio $\geq 2$. Using this older definition, the prevalence of positional dependency in milder OSA patients (AHI 10-19.9/h) in a sleep center by Arie Oksenberg in Israel is 65.1\% [7], and $87 \%$ in milder OSA patients (AHI $=5-19.9)$ in Korea [19]. However, with the recent definition of positional dependency as used (including normalized nonsupine $\mathrm{AHI}$ ), the prevalence of positional dependency is $36 \%$ of all OSA patients reported by Heinzer et al. [11], and 49.5\% of mild OSA patients as well as reported by Mador et al. [18]. The prevalence of positional dependency in our mild OSA patients was $76.8 \%$, higher than the prevalence reported by Mador et al. [18] possibly due to the patients in our study were highly selected because we only collected patients keeping followed up in our hospital.

The treatment rate of our mild OSA patients, and even the positional mild OSA patients, was very low (12.9\% for mild OSA, and also $12.9 \%$ for positional mild OSA). The follow-up rate was also low, as only $36.1 \%$ of mild OSA patients returned for the follow-up. In Taiwan, there are 60 sleep centers providing enough PSG examination resources. PSG examinations of the Taiwan National Health Insurance Administration cost only USD 155. The cheap and convenient PSG examination resources resulted in the increasing diagnosis of patients with mild OSA. Our sleep center has been continuously committed to OSA education for all medical staff in the past 15 years since its foundation, resulting in increasing numbers of patients with mild OSA receiving PSG screening, regardless of symptoms and cardiovascular comorbidities. These screened patients had less OSA symptoms, were less willing of active treatment, and inclined to refuse close follow-up. These explained the higher prevalence, the lower treatment rate, and the lower follow-up rate of mild OSA patients in our sleep center. Our present study presented real-world conditions based on our sleep center data. Many of these mild OSA patients did not receive a followup PSG not only because they did not agree but also because they were not advised close follow-up. Clinicians might not consider mild OSA as a critical problem and 
typically advise patients to control weight and only return in case of symptom deterioration. Confirming previous studies, our finding suggested that mild OSA is progressive, regardless of positional preponderance $[12,14,20$, 21]. There was an association between increased OSA severity and $\mathrm{BP}$ even in patients with positional mild OSA. This suggests that clinicians and sleep specialists should increase awareness regarding positional mild OSA. There is a need for attention regarding active treatment and follow-up for patients with mild OSA, regardless of positional dependency.

Mild OSA tends to worsen over time [12-14], which also holds true for positional mild OSA as shown in our present study. In an earlier study, we also documented that positional OSA progressed over time regardless of positional dependency [10], thus suggesting that active treatment for OSA regardless of positional dependency is important.

In 2012, Oksenberg et al. [8] found the dynamic interaction between increasing weight and positional dependency and that weight reduction had benefits changing from nonpositional to positional dependency. In 2020, Oksenberg et al. also reported $29.6 \%$ of patients with positional OSA converted to nonpositional OSA after an average 6.6 years of follow-up [15]. In our study, we also found that positional dependency disappeared over time even in mild OSA patients. In the report by Oksenberg et al. [7], the most significant predictors for positional dependency were sequentially higher $\mathrm{AHI}$, higher $\mathrm{BW}$, and older age [7], but only BW was associated with the loss of positional dependency in our study because we just focused on mild OSA patients who were younger and had lower AHI. Weight loss had beneficial effects of positional dependency conversion [7] suggesting the importance of weight control in positional mild OSA patients.

In OSA patients, sleep apnea events result in repeated arousal from deep sleep to superficial sleep; therefore, the $\mathrm{N} 1$ sleep stage implied that superficial sleep will be increased with increasing AHI. In our study, we also found that increasing N1 sleep stage was associated with AHI worsening.

The relationship between increasing BW and OSA progression is well documented $[7,14,22,23]$. Reduction of BW could result in the improvement of OSA severity [7]. In our study, we also documented increasing weight was the most valuable predictor of increasing AHI among mild OSA patients and positional mild OSA patients. The follow-up duration (5.1 \pm 3 years) was also associated with AHI increases in a previous report [14], but we did

Natural Course of Positional Mild

Obstructive Sleep Apnea not find this result, which might be due to the follow-up duration in our study being only $32.0 \pm 27.6$ months, obviously shorter. Here, it is worth mentioning that our result showed mild OSA is progressive even in short followup time regardless of positional dependency, suggesting more intensive follow-up is warranted for mild OSA regardless of positional dependency.

Although BW reduction is important for OSA, this is difficult in this time of increasing prevalence of obesity [24]. Since BW maintenance or reduction is part of the standard treatment recommended for positional mild OSA, more aggressive BW reducing strategies and more intensive monitoring should be considered.

Previous studies reported the factors of BP increases in OSA patients were follow-up time, increasing weight, and increasing AHI $[1,25,26]$. In the report of Vallée et al. [25], they found that dynamic BW changes, instead of the static view expressed by BMI, appear more predictive of $\mathrm{BP}$ changes. In men, weight variations $>+1 \mathrm{~kg} /$ year are associated with a $5.5-\mathrm{mm} \mathrm{Hg}$ increase in $\mathrm{sBP}$ and with a $1.8 \mathrm{~mm} \mathrm{Hg}$ increase in diastolic BP (dBP). In women, $>+1$ $\mathrm{kg} /$ year is associated with a $3.3-\mathrm{mm} \mathrm{Hg}$ increase in sBP and a $1.0 \mathrm{~mm} \mathrm{Hg}$ increase in $\mathrm{dBP}$. In the Chinese population, He et al. found that OSA severity was associated with increasing $\mathrm{BP}$, and that $\mathrm{dBP}$ is inclined to rise faster and not parallel with increasing sBP [26]. In our study, we only found increasing sBP but not increasing $\mathrm{dBP}$. Elevated BP was noted in mild OSA patients not treated with $\mathrm{CPAP}$, and it took over a 2-year period of CPAP treatment to lower the elevated BP [1]. In our study, the predictor for increasing sBP was only age at follow-up, but not $\mathrm{AHI}$ and $\mathrm{BW}$. This might be due to $\mathrm{BP}$ control being multifactorial and the lack of control of confounders in this study.

Active treatment for mild OSA and even positional mild OSA is important to prevent its progression. PT is effective in improving sleep architecture and symptoms for positional OSA patients, but the long-term compliance of PT is low and is difficult to be monitored $[9,27$, 28]. In our report, we had shown positional mild OSA progressed over time, mainly due to weight increasing, and resulting in increasing BP and ANS dysregulation. $B W$ reduction will be effective to prevent the progression of positional mild OSA. However, BW reduction is often difficult in this era; only about $20 \%$ of patients in the general population could achieve success at long-term weight loss maintenance [29]. Since BW reduction is difficult and the long-term compliance of PT is poor, more active treatments and close follow-up for positional mild OSA are necessary. 
OSA affects ANS regulation in a dose-dependent manner, and OSA treatment results in improvement of ANS dysregulation $[5,30]$. Our findings also showed similar effects on ANS dysregulation in patients with progressive mild OSA.

\section{Conclusion}

In patients with positional mild OSA, the disease severity, sleep quality, BP, and ANS regulation worsened over time, while the positional dependency disappeared after the follow-up. Increased weight was the most valuable predictor for OSA progression and loss of positional dependency. Our findings emphasize the importance of BW control and consistent follow-up for positional mild OSA.

\section{Limitations}

This study has several limitations. First, the lack of nasal pressure transducers could have led to misclassification of patients as having milder OSA forms. Second, we used a trunk-position sensor during respiratory inductance plethysmography without a head position sensor, which could have led to misjudgment of sleep positions. Third, the small number of cases might have resulted in selection bias. Fourth, although this retrospective cohort study design may be representative of a real-world situation, the small number of included patients might not represent all patients with mild OSA. Finally, most patients with mild OSA did not return or refused the followup; therefore, it was difficult to determine whether they had received other treatments or if their mild OSA worsened over time. There is a need for larger, well-controlled prospective studies.

\section{Acknowledgement}

We would like to thank Editage (www.editage.com) for English language editing.

\section{Statment of Ethics}

This study was approved by the Institutional Review Board of Taipei Tzu Chi Hospital (IRB protocol No. 07-XD-049). Informed consent was waived due to the retrospective study design.

\section{Conflict of Interest Statement}

The authors have no conflicts of interest to declare.

\section{Funding Sources}

This study was supported by a grant from the Taipei Tzu Chi Hospital, Buddhist Tzu Chi Medical Foundation (TCRD-TPE108-RT-3).

\section{Author Contributions}

Mei-Chen Yang, Hsien-Chang Wu, and Wei-Hsiu Chang designed the study and collected patients' data. Chou-Chin Lan and Yao-Kuang Wu analyzed the data. Mei-Chen Yang and Wei-Hsiu Chang prepared the manuscript. Yao-Kuang Wu, Chou-Chin Lan, and Hsien-Chang Wu collected references. Mei-Chen Yang and Chou-Chin Lan made critical revisions to the manuscript. MeiChen Yang applied the IRB approval and the funding for publication.

\section{References}

1 Jaimchariyatam N, Rodriguez CL, Budur K. Does CPAP treatment in mild obstructive sleep apnea affect blood pressure? Sleep Med. 2010 Aug;11(9):837-42.

2 Collen J, Lettieri C, Wickwire E, Holley A. Obstructive sleep apnea and cardiovascular disease, a story of confounders!. Sleep Breath. 2020 Jan 9;24(4):1299-313.

3 Dredla BK, Castillo PR. Cardiovascular consequences of obstructive sleep apnea. Curr Cardiol Rep. 2019 Nov;21(11):137.

4 McNicholas WT, Bonsignore MR, Lévy P, Ryan S. Mild obstructive sleep apnoea: clinical relevance and approaches to management. Lancet Respir Med. 2016 Oct;4(10): 826-34.
5 Palma JA, Iriarte J, Fernandez S, Alegre M, Valencia M, Artieda J, et al. Long-term continuous positive airway pressure therapy improves cardiac autonomic tone during sleep in patients with obstructive sleep apnea. Clin Auton Res. 2015 Aug;25(4):225-32.

6 Lurie A. Obstructive sleep apnea in adults. Hemodynamic and autonomic changes in adults with obstructive sleep apnea. Adv Cardiol. 2011;46:171-95.

7 Oksenberg A, Silverberg DS, Arons E, Radwan $\mathrm{H}$. Positional vs nonpositional obstructive sleep apnea patients: anthropomorphic, nocturnal polysomnographic, and multiple sleep latency test data. Chest. 1997 Sep;112(3): 629-39.
8 Oksenberg A, Dynia A, Nasser K, Gadoth N Obstructive sleep apnoea in adults: body postures and weight changes interactions. J Sleep Res. 2012 Aug;21(4):402-9.

9 de Vries GE, Hoekema A, Doff MH, Kerstjens HA, Meijer PM, van der Hoeven JH, et al. Usage of positional therapy in adults with obstructive sleep apnea. J Clin Sleep Med. 2015 Jan;11(2):131-7.

10 Huang YC, Lin CY, Lan CC, Wu YK, Lim CS, Huang $\mathrm{CY}$, et al. Comparison of cardiovascular co-morbidities and CPAP use in patients with positional and nonpositional mild obstructive sleep apnea. BMC Pulm Med. 2014 Sep;14:153. 
11 Heinzer R, Petitpierre NJ, Marti-Soler H, Haba-Rubio J. Prevalence and characteristics of positional sleep apnea in the HypnoLaus population-based cohort. Sleep Med. 2018 Aug; 48:157-62.

12 Sahlman J, Pukkila M, Seppä J, Tuomilehto H Evolution of mild obstructive sleep apnea after different treatments. Laryngoscope. 2007 Jun;117(6):1107-11.

13 Oksenberg A, Goizman V, Eitan E, Nasser K, Gadoth N, Leppänen T. Obstructive sleep apnea: do positional patients become nonpositional patients with time? Laryngoscope. 2020 Sep;130(9):2263-8.

14 Berger G, Berger R, Oksenberg A. Progression of snoring and obstructive sleep apnoea: the role of increasing weight and time. Eur Respir J. 2009 Apr;33(2):338-45.

15 Chen NH, Johns MW, Li HY, Chu CC, Liang $\mathrm{SC}$, Shu YH, et al. Validation of a Chinese version of the epworth sleepiness scale. Qual Life Res. 2002 Dec;11(8):817-21.

16 Malik M, Bigger JT, John Camm A, Kleiger R, Malliani A, Moss A, et al. Heart rate variability. standards of measurement, physiological interpretation, and clinical use. Task force of the European society of cardiology and the North American society of pacing and electrophysiology. Eur Heart J. 1996 Mar;17(3): 354-81.

17 Oksenberg A, Gadoth N. Positional therapy is a valuable treatment alternative for obstructive sleep apnea. J Transl Int Med. 2019 Jul; $7(2): 84-5$.
18 Mador MJ, Kufel TJ, Magalang UJ, Rajesh SK, Watwe V, Grant BJ. Prevalence of positional sleep apnea in patients undergoing polysomnography. Chest. 2005 Oct;128(4):2130-7.

19 Mo JH, Lee CH, Rhee CS, Yoon IY, Kim JW. Positional dependency in Asian patients with obstructive sleep apnea and its implication for hypertension. Arch Otolaryngol Head Neck Surg. 2011;137(8):786-90.

20 Pendlebury ST, Pépin JL, Veale D, Lévy P. Natural evolution of moderate sleep apnoea syndrome: significant progression over a mean of 17 months. Thorax. 1997;52(10): 872-8.

21 Tuomilehto H, SeppäJ, Uusitupa M, Peltonen M, Martikainen T, Sahlman J, et al. The impact of weight reduction in the prevention of the progression of obstructive sleep apnea: an explanatory analysis of a 5-year observational follow-up trial. Sleep Med. 2014;15(3):32935.

22 Wolk R, Shamsuzzaman AS, Somers VK. Obesity, sleep apnea, and hypertension. Hypertension. 2003 Dec;42(6):1067-74.

23 Leppanen T, Kulkas A, Mervaala E, Toyras J. Increase in body mass index decreases duration of apneas and hypopneas in obstructive sleep apnea. Respir Care. 2019 Jan;64(1):7784.

24 Wang Y, Beydoun MA, Min J, Xue H, Kaminsky LA, Cheskin LJ. Has the prevalence of overweight, obesity and central obesity levelled off in the United States? Trends, patterns, disparities, and future projections for the obesity epidemic. Int J Epidemiol. 2020 Jun;49(3):810-23.
25 Vallée A, Perrine AL, Deschamps V, Blacher J, Olié V. Relationship between dynamic changes in body weight and blood pressure: the ESTEBAN survey. Am J Hypertens. 2019 Sep;32(10):1003-12.

26 He QY, Feng J, Zhang XL, Liang ZA, Huang SG, Kang J, et al. Elevated nocturnal and morning blood pressure in patients with obstructive sleep apnea syndrome. Chin Med J. 2012 Oct;125(10):1740-6.

27 Jokic R, Klimaszewski A, Crossley M, Sridhar G, Fitzpatrick MF. Positional treatment vs continuous positive airway pressure in patients with positional obstructive sleep apnea syndrome. Chest. 1999 Mar;115(3):771-81.

28 Ravesloot MJL, White D, Heinzer R, Oksenberg A, Pépin JL. Efficacy of the new generation of devices for positional therapy for patients with positional obstructive sleep apnea: a systematic review of the literature and metaanalysis. J Clin Sleep Med. 2017 Jun;13(6): 813-24.

29 Wing RR, Phelan S. Long-term weight loss maintenance. Am J Clin Nutr. 2005 Jul; 82(Suppl 1):222S-5S

30 Gong X, Huang L, Liu X, Li C, Mao X, Liu W, et al. Correlation analysis between polysomnography diagnostic indices and heart rate variability parameters among patients with obstructive sleep apnea hypopnea syndrome. PLoS One. 2016 Jun 2;11(6):e0156628. 